\title{
Dental Clenching: A Way to Improve Body Stability in Subject Suffering From Whiplash Injury. Part 2: Patients with Malocclusion
}

\author{
Maurizio Stefano Giacomello* \\ University of Milan-Bicocca, Italy
}

Submission: December 08, 2015; Published: February 02, 2016

*Corresponding author: Maurizio S Giacomello, School of Medicine and Surgery, University of Milan-Bicocca, Viale Casati 51 I-20867 Caponago, Italy, Tel: +393358332943; Email: maurizio.giacomello@unimib.it

\begin{abstract}
Aim: Aim of this study is analyze the variations on the postural stability in patients with previous whiplash with different conditions of dental occlusion.

Materials and Methods: 66 adult patients suffering from masticatory muscles pain caused by clenching during sleepwith previous experience of whiplash were selected for this study.

Criteria for exclusion: Vestibular or neurologic pathologies, use of prescription drugs or psychotropic substances. The patients have been divided into two groups: 36 adult patients with normal occlusion (good alignment, 1st class or mild 2nd class deep bite for mandibular retrusion) constituted the group A, while 30 adult patients with previous whiplash and malocclusion (cross bite, mandibular shift, bad alignment, edentulous spaces) constituted the group B. To simulate the conditions of sleep (with a reduction of proprio- and esteroceptive inputs for postural management performed by CNS), we used the postural Romberg analysis (feet together, closed eyes) on a stabilometric platform changing occlusal parameters only, under the following conditions: 1) in occlusalrest position, 2) in centricocclusion, 3) in clenching, 4) with occlusal disengage with cotton rolls and 5) in clenching on cotton rolls.
\end{abstract}

Time of each test: 15 seconds. It was performed a statistical analysis with T-test comparing between them the mean of all tests.

Results: For the Group A the stabilometry data (postural ball and ellipse of COG - center of gravity) show better postural performances in centric occlusion and clenching in centric occlusion, and a worsening in all tests with occlusal disengage (with statistically significant difference - $\mathrm{p}$ <.05). For the group B (with malocclusion) the stabilometry data show better postural results with occlusal disengage (in centric and in clenching with cotton rolls between the arches), and a worsening in all tests when the patients are in mandibular rest position, in centric occlusion or in clenching in centric occlusion (with statistically significant difference - $p<0.05$ ).

Conclusion: Postural instability caused by whiplash in creases muscle stone and dental clenching as a compensatory effects, with onset of facial pain due to muscula rtension. In patients with good occlusion an occlusal disengage (like the cottonrolls used in this work, or an occlusal splint to reduce clenching) induces a postural worsening in these patients, while centric occlusion increases the stability. So these patients could begin to clench during the sleep to compensate an unstable posture for the previous whiplash: in fact the absence of the stabilizing effect of the vision and the podalicload (during the sleeplying down) makes these subjects more unstable. During the sleep this condition induces a compensative response of the CNS, with increase of the body's muscular activity with clenching of the teeth. For these patients the use of a splint can increase their instability because it changes the structure that stabilize their postural system after the whiplash: the stomatognathic apparatus. In case of malocclusion (group B) the postural instability caused by whiplash increases muscular tone and the dental clenching increases the negative effects of the malocclusion on the stomatognathic apparatus, with onset of facial pain and a worsening of TMJ health. An occlusal disengage (like an occlusal splint) induces better postural performances because it removes the negative effects of the malocclusion on the masticatory muscles, on the TMJs giving to the postural system a new, better point of stabilization. In these patients the malocclusion becomes sources of postural instability and a cause of postural worsening in association to the effects of whiplash injury. These results suggest that stomatognathic organ has a new physiological function: the stabilization of the body posture.

Keywords: Posture; Whiplash; Dental Occlusion; Clenching; Malocclusion; Cranio-Mandibular Relationship; Stabilometry; TMD; TemporoMandibular Disorders 


\section{Introduction}

Oneoftheconditionsthat traditionally requiresagnathological treatment is represented by dental clenching, a condition that is often considered as an expression of a psychogenic tension state and therefore mistook with bruxism. Patients suffering from this para physiological condition they often complain morning pain around the cranio-cervical-mandibular muscles, which is a consequence of a prolonged contraction state of the muscles of jaw raising [1-5]. It is a condition that is recognized in different causes, and that is often appears after a cervical whiplash injury, this condition determines a heavy modification of the patients' postural performances [6-9]. The purpose of this second part it consists into instrumentally evaluate the importance of the stomatognathic system in the postural stabilization of patients with malocclusion who had suffered from a traumatic event, such as the cervical spine distortion, known as whiplash, which typically causes consequences of a postural kind.

\section{Materials and Methods}

30 adult patients had been selected (19 males and 11 females, average age 34.77 aa, St. dev. 9.33 aa, range 20-51) from a group of 66 suffering from nocturnal dental clenching with existing whiplash. Criteria for exclusion: good occlusion, vestibular or neurologic pathologies, use of prescription drugs or psychotropic substances. The patients of this second group presented a malocclusion characterized by dental crowding on both arches, missing teeth (above all in the posterior part of the arches), cross bites, mandibular shift or accentuated asymmetry in curves of Spee and Wilson, or in second or third skeletal class: the common trait was an altered cranio-mandibular space rapport (shift CO-CR). The postural evaluation done on stabilometric platform (Sensor Medica, Roma, Italy) has analyzed the patients with the Romberg postural test (patients with feet together and closed eyes) by a protocol created by Dr. Giacomello and therefore it carries his name (Giacomello's Protocol). It includes the implementation of five different tests during which it exclusively affects the dental arches relationships: 1) in rest position, 2) in centric occlusion (habitual or maximum intercuspidation), 3) clenched jaw with habitual occlusion, 4) with occlusal disengage between the arches (e.g. two cotton rolls between the arches), 5) clenched jaw with cotton rolls in between the arches. Each test lasted 15 seconds. Hence we proceeded to evaluate the stabile metric parameters obtained from the various tests (length of the postural ball length and ellipse area), by performing a statistical comparison via Student T-test of paired facts for both postural variables in the tested conditions (Figure1\&2). We have then confronted the single differential test conditions between the two groups and evaluating the statistical significance through the Student T-test (per groups with a dissimilar variation).
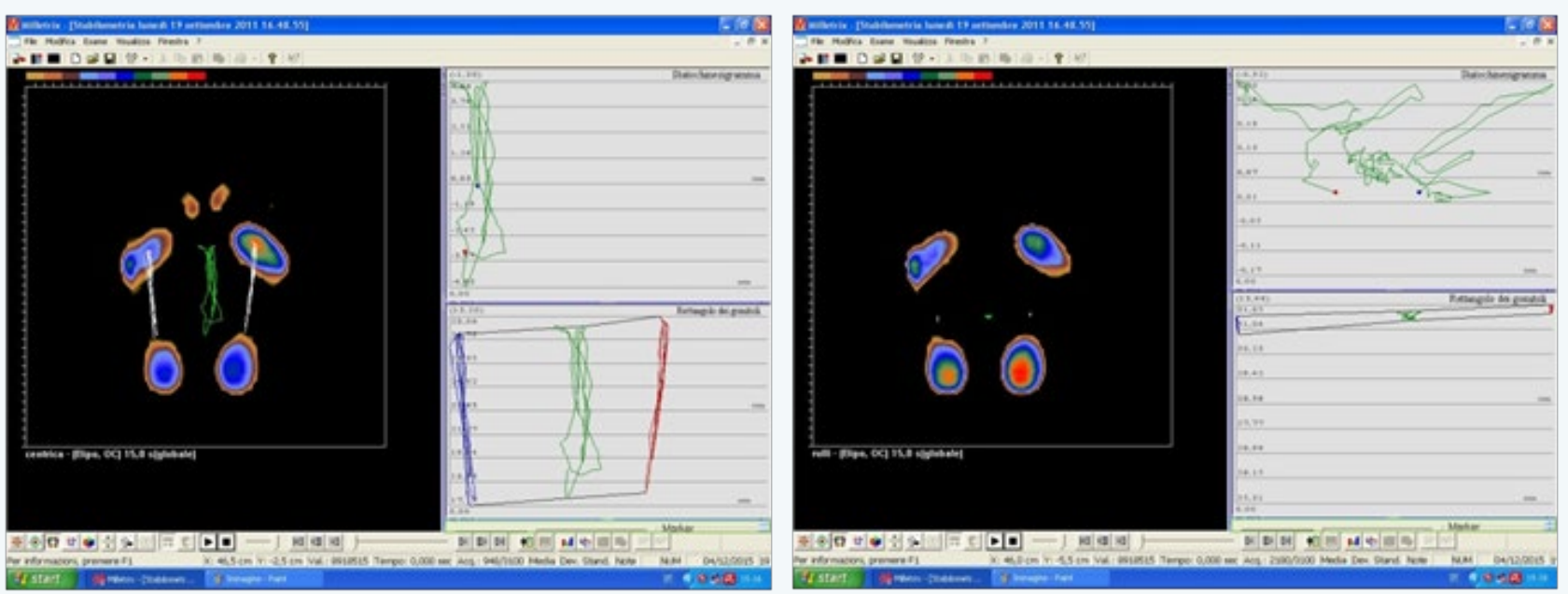

Figure 1 (on the left) and 2 (on the right): Analysis of the podalic load and the behavior of the Center of Gravity (green line) in centric occlusion (left) and with occlusal disengage (cotton rolls among the arches) in subject with malocclusion: it is evident the increase of body stability in the second image, measured by the length of the two green lines (behavior of the center of gravity).

\section{Results}

From a stabilometric point of view we considered as values to analyze the postural ball length and the ellipse area, or else the work and the effort made by the body to maintain an erected posture and a good balance. The postural ball length is graphically represented by a broken line which is situated inside the supporting polygon, and it corresponds to the rocking movements made by the projection of the body bary centre to the ground during the exam acquisition. It is the expression of the behavior of the body bary centre itself. The final values obtained during the various tested conditions have been reported in Table 1 (postural ball length in $\mathrm{mm}$ ) and in Table 2 (postural ellipse area in $\mathrm{mm}^{2}$ ). 
Table1: Results of the postural ball in different tested conditions for patients with malocclusion (in millimeters).

\begin{tabular}{|c|c|c|c|c|c|}
\hline Post ball & Rest & Centric & Clench & Rolls & Cl rolls \\
\hline 1 & 126,76 & 118,24 & 143,43 & 112,96 & 71,00 \\
\hline 2 & 183,24 & 138,35 & 115,40 & 72,89 & 56,19 \\
\hline 3 & 23,59 & 29,21 & 27,62 & 20,74 & 33,94 \\
\hline 4 & 57,11 & 45,59 & 40,93 & 53,99 & 74,79 \\
\hline 5 & 87,61 & 108,66 & 171,67 & 80,93 & 76,51 \\
\hline 6 & 64,12 & 92,32 & 106,13 & 78,87 & 101,05 \\
\hline 7 & 57,11 & 45,59 & 53,99 & 40,39 & 74,79 \\
\hline 8 & 43,57 & 37,23 & 32,84 & 44,59 & 58,57 \\
\hline 9 & 276,94 & 239,52 & 241,47 & 170,78 & 253,98 \\
\hline 10 & 1712,50 & 563,36 & 867,97 & 352,48 & 477,88 \\
\hline 11 & 131,90 & 111,35 & 159,45 & 108,32 & 68,13 \\
\hline 12 & 179,63 & 143,38 & 131,35 & 68,63 & 63,95 \\
\hline 13 & 25,45 & 35,28 & 37,13 & 18,84 & 29,89 \\
\hline 14 & 62,98 & 43,21 & 42,56 & 61,78 & 69,31 \\
\hline 15 & 93,30 & 114,89 & 193,26 & 77,26 & 74,92 \\
\hline 16 & 60,19 & 102,93 & 131,45 & 81,98 & 118,93 \\
\hline 17 & 59,34 & 51,92 & 68,29 & 36,82 & 89,99 \\
\hline 18 & 41,89 & 32,25 & 48,19 & 49,91 & 68,83 \\
\hline 19 & 284,58 & 247,89 & 247,92 & 166,91 & 171,93 \\
\hline 20 & 1845,23 & 636,83 & 902,73 & 371,98 & 304,50 \\
\hline 21 & 473,29 & 384,69 & 288,28 & 281,57 & 255,44 \\
\hline 22 & 474,75 & 362,61 & 486,19 & 312,98 & 356,40 \\
\hline 23 & 413,38 & 310,33 & 297,14 & 270,17 & 204,86 \\
\hline 24 & 246,93 & 317,13 & 261,32 & 223,06 & 167,69 \\
\hline 25 & 276,94 & 239,52 & 241,47 & 170,78 & 253,98 \\
\hline 26 & 470,38 & 380,99 & 284,48 & 275,36 & 247,35 \\
\hline 27 & 477,45 & 370,89 & 492,73 & 317,22 & 361,17 \\
\hline 28 & 403,74 & 300,28 & 287,98 & 260,04 & 394,86 \\
\hline 29 & 257,39 & 328,97 & 273,94 & 235,64 & 279,14 \\
\hline 30 & 262,84 & 225,27 & 227,94 & 166,94 & 238,06 \\
\hline AVERAGE & 305,80 & 205,29 & 230,18 & 152,83 & 169,93 \\
\hline ST DEV & 429,01 & 162,57 & 216,17 & 109,94 & 123,86 \\
\hline
\end{tabular}

Table 2: Results of the extension of the postural ellipse in different tested conditions for patients with malocclusion (in square millimeters).

\begin{tabular}{|c|c|c|c|c|c|}
\hline Ellipse & Rest & Centric & Clench & Rolls & Cl rolls \\
\hline 1 & 798,88 & 383,27 & 1040,35 & 527,42 & 91,56 \\
\hline 2 & 307,18 & 125,92 & 247,24 & 219,92 & 78,23 \\
\hline 3 & 6,06 & 11,62 & 6,22 & 3,36 & 17,38 \\
\hline 4 & 82,06 & 40,16 & 40,39 & 105,88 & 430,11 \\
\hline 5 & 299,24 & 461,31 & 3150,84 & 125,40 & 187,82 \\
\hline 6 & 102,42 & 103,91 & 242,49 & 131,60 & 181,65 \\
\hline 7 & 57,11 & 45,59 & 40,39 & 53,99 & 74,79 \\
\hline 8 & 53,83 & 36,09 & 19,65 & 40,06 & 90,21 \\
\hline 9 & 595,23 & 456,05 & 741,29 & 545,00 & 561,65 \\
\hline 10 & 18706,57 & 2641,01 & 5659,12 & 1348,70 & 1332,07 \\
\hline 11 & 790,33 & 373,12 & 997,35 & 520,92 & 89,46 \\
\hline 12 & 300,88 & 120,02 & 240,24 & 227,02 & 80,25 \\
\hline 13 & 5,06 & 9,98 & 7,82 & 5,76 & 19,46 \\
\hline 14 & 78,06 & 42,16 & 44,09 & 109,98 & 427,91 \\
\hline 15 & 295,20 & 467,91 & 3232,79 & 130,54 & 188,52 \\
\hline 16 & 99,22 & 100,27 & 250,59 & 125,58 & 179,05 \\
\hline 17 & 55,01 & 47,99 & 44,79 & 50,32 & 76,89 \\
\hline 18 & 50,83 & 37,10 & 21,55 & 43,86 & 88,31 \\
\hline 19 & 590,23 & 460,05 & 736,18 & 573,94 & 458,45 \\
\hline 20 & 17506,77 & 2543,00 & 5599,89 & 1329,44 & 1298,97 \\
\hline 21 & 1141,19 & 1313,34 & 875,90 & 523,05 & 593,77 \\
\hline 22 & 731,19 & 309,47 & 630,12 & 303,23 & 353,80 \\
\hline 23 & 674,31 & 564,82 & 579,49 & 607,69 & 549,83 \\
\hline 24 & 831,09 & 1069,97 & 781,38 & 489,90 & 504,12 \\
\hline 25 & 595,23 & 456,05 & 741,29 & 545,00 & 561,65 \\
\hline 26 & 1127,92 & 1301,33 & 861,15 & 509,29 & 579,34 \\
\hline 27 & 744,76 & 322,68 & 643,10 & 316,54 & 366,47 \\
\hline 28 & 670,94 & 560,36 & 575,94 & 601,35 & 542,83 \\
\hline 29 & 820,46 & 1055,46 & 773,24 & 480,50 & 790,95 \\
\hline 30 & 602,65 & 464,62 & 753,89 & 559,34 & 571,03 \\
\hline AVERAGE & 1624,00 & 530,82 & 985,96 & 371,82 & 378,88 \\
\hline ST DEV & 4496,45 & 672,67 & 1477,77 & 339,86 & 337,73 \\
\hline
\end{tabular}


The average values related to the postural ball length have been (in $\mathrm{mm}$ ).

$\begin{array}{llll}1 & \text { In Rest Position } & 305.80 & \text { St. dev. } 429.01 \\ 2 & \text { In Centric Occlusion } & 205.29 & \text { St. dev. } 162.57 \\ 3 & \text { Clenching In Centric Occlusion } & 230.18 & \text { St. dev. } 216.17 \\ 4 & \text { With Occlusal Disengage } & 152.83 & \text { St. dev. } 109.94 \\ 5 & \text { Clenching On Cotton Rolls } & 169.93 & \text { St. Dev. } 123.86\end{array}$

The average values related to the postural ellipse have been (in $\mathrm{mm}^{2}$ ).
1 In Rest Position
1624.00 St. dev. 4496.45
2 In Centric Occlusion: Average
530.82 St. dev. 672.67
3 Clenching In Centric Occlusion
4 With Occlusal Disengage
985.96
St. dev. 1477.77
5 Clenching on cotton rolls
371.82
St. dev. 339.86
378.88
St. dev. 337.73

Hence we proceeded to confront the statistical analysis of the obtained averages via Student T-test with paired facts. Nonetheless to give more meaning to the comparison, the following obtained average facts had been considered in the following conditions (for postural ball length).

$\begin{array}{lll}1 & \text { Rest Position - Centric Occlusion } & 0.074 \\ 2 & \text { Rest Position - Clenching } & 0.085 \\ 3 & \text { Centric Occlusion - Clenching } & 0.125 \\ 4 & \text { Centric Occlusion - Cotton Rolls } & 0.0001(\mathrm{p}=0.0001) \\ 5 & \text { Clenching - Cotton Rolls } & 0.003(\mathrm{p}<0.01) \\ 6 & \text { Clenching - Clenching On Rolls } & 0.020(\mathrm{p}<0.05) \\ 7 & \text { Cotton Rolls - Clenching On Rolls } & 0,074\end{array}$

Unlike what we observed on patients with normal occlusion, in this second group the postural ball length, expressing the work performance from the postural system to maintain its physiological balance, it results statistically minor in the test conditions done with the occlusal disengage, and especially when comparing the tests in habitual centric (media: $205.29 \mathrm{~mm}$ ) and with cotton rolls in between the dental arches (media: 152.83 $\mathrm{mm})$ with a high statistical significance $(\mathrm{p}=0.0001)$. Therefore we gathered that the dental intercuspidation represents in this patients a strong postural destabilizing condition, while placing in between the arches two cotton rolls (occlusal disengages) the postural system improves with a reduction of the work performed and a lower energy consumption: in these patients the occlusal disengage (which simulates a resin splint) guarantees better postural performances. Also during the other statistical comparisons done between the conditions with or without occlusal disengage, they show that in these patients the intercuspidation is source of a strong postural instability, and such phenomenon diminishes when placing cotton rolls in between the dental arches to improve the occlusal stability and the intercuspidation. Similarly for the postural ellipse (expressing the body stability) we obtained.

$\begin{array}{lll}1 & \text { Rest Position - Centric Occlusion } & 0.138 \\ 2 & \text { Rest Position - Clenching } & 0.299 \\ 3 & \text { Centric Occlusion - Clenching } & 0.019(\mathrm{p}<0.05) \\ 4 & \text { Centric Occlusion - Cotton Rolls } & 0.038(\mathrm{p}<0.05) \\ 5 & \text { Clenching - Cotton Rolls } & 0.012(\mathrm{p}<0.05) \\ 6 & \text { Clenching - Clenching On Rolls } & 0.014(\mathrm{p}<0.05) \\ 7 & \text { Cotton Rolls - Clenching On Rolls } & 0,814\end{array}$

Also for the postural ellipse area, expressing the body stability, we have observed better postural during the test conditions with the use of occlusal disengage, while the conditions of the analysis done in rest position and clenched they result to have significantly worse, in part because of the remarkable postural instability caused by the whiplash, and in part due to the malocclusion which negative effects are exasperated by the dental clenching tendency: in other terms clenching ones teeth worsens the whole dysfunction due to the already present malocclusion. Lastly the comparison of testing with rolls and clenching with rolls shows a worsening, therefore not significant, of the postural performances in subjects with malocclusion: such should be attributed, in our opinion, to the tendency to an asymmetrical muscular activation while clenching, which is because it's a condition structured over time and that requires time to be modified: in other terms a few minutes of the test performance are not enough to modify the state of maximal muscle activation, as it happens during clenching, that is why they normally are performing under asymmetrical and incongruous functionality schemes, and for it to modify it requires a longer time in comparison to what was observed during the condition over rolls. In the end the comparison in between the two groups shows a major postural instability in the patients belonging to the second group in the conditions of rest position, centric and clenching over centric with statically significant differences $(\mathrm{p}<0.05)$, while in the two tests done with the cotton rolls such difference was reduced drastically to the point of not being significant any longer.

\section{Discussion}

The whiplash traumatic pathology is the consequence of a subsequent trauma in which a abrupt acceleration and the following deceleration will cause the violent stretching and bending of the neck [10-13]. The social incidence of the whiplash needs to be evaluated not only in diagnostic-therapeutically terms, but also epidemiological; such lesion in fact it represents one of the major subsequences of trauma from road accidents; additionally is one of the principle causes of postural disturbance of traumatic origin [14-18]. Many patients that have suffered from this trauma they will present with time gnathologic problems (coronal fissures and fractures, parodonthal occlusal 
trauma, TMJ pain, or alterations of the articular morphology, muscle pains with possible arising of headaches) and tendency to dental arches clenching, phenomenon which usually happens during ones sleep, accompanied by cranio-facial pain. It needs to be pointed out that patients recognize to have a tendency for clenching in a period of time subsequent to the trauma, and its appearance happens about 20-30 days after having suffered from whiplash. If in group 1 we can state that the clenching worsens the algic symptomatology in charge of the cranio-cervical muscle, which was already suffering from the post-traumatic period; for the patients from group 2 the stomatognathic structures appear to be already suffering and the trauma worsens an already dysfunctional condition: indeed clenching causes an excess of TMJ pressure aggravating and resulting with the manifestation of a algic-dysfunctional pathology (indirect lesive action), which was already present and apparently compensated for. It needs to be mentioned that this articulation might be already suffering due to the trauma (direct lesive action) due to the typical abrupt whiplash cranio-cervical movement $[19,20]$. The postural facts obtained during the present study show therefore the extreme importance of the stomatognathic system in the neuro physiological management of the postural: in fact the patients with malocclusion present clearly worse postural conditions in comparison to the patients from group 1 (without malocclusion), with statistically significant differences (Table 2); moreover the differences in between the two groups of patients leans to reduce noticeably in the tests done with occlusal disengage by improving the performances viewed in group 2 (in comparison to the normal occlusion tests), and by a visible worsening of group 1 . The postural results of group 2 are definitely worse in tests done with normal occlusion (tests 1-3), while in tests 4 and 5 (with occlusal disengage) they are definitely better; moreover these patients show a much more accentuated postural instability than of those from group 1 (post whiplash but without malocclusion) and this fact tends to reduce itself drastically once an occlusal disengage is inserted in between the dental arches to neutralize the malocclusion, with a very high statistical significance. Hence the result is that in some forms of malocclusion the stomatoganthic system represents an important source of postural instability which worsens with whiplash. In these cases, from a therapeutically point of view, it needs a combined approach (gnathologic and postural) in order to solve both causes of the disorder: as opposed to the patients free of malocclusion, which through the occlusal stability they try to obtain a postural stabilization and for whom the use of the bite could be source of further instability (and therefore cause more clenching), in the patients with malocclusion the use of a resin plaque (for disengage or repositioning), it results in being fundamental to create new strategies for postural stability in association with other therapies of a postural character. The condition of centric occlusion or maximum intercuspidation represents an important reference in the neuro physiological management of the posture, or else it represents a fundamental base to guarantee the body stability (in case of normal occlusion) or to worsen the instability (in case of malocclusion) in subjects with poor postural stability due to other causes such as whiplash. We can therefore deduce that in the first group the stomatognathic system becomes victim of a dysfunctional scheme that recognizes the trauma (whiplash) the causing agent, while in the second group the necessity to clench their teeth amplifies the negative consequences, yet already present, of the malocclusion with sufferings from the structures that are part of the stomatognathic system (teeth, parodonthal, but mostly TMJ and muscles). Additionally this study allows to high light the importance of this type of approach in medical-legal evaluations to determine the real entity of the damage from whiplash over the stomatognathic system. In fact in case of malocclusion the dysfunctional scene could be well compensated for, a condition that alters when exposed to a traumatic event such as whiplash, which could cause an algic dysfunctional pathology which recognizes in the malocclusion the causing element along with the trauma effects.

\section{Conclusion}

The present work represents the second part of a more complex project which objective is to understand the importance of dental occlusion in within the postural system. This is why we analyzed subjects that presented a postural instability trauma originated (in the event of post whiplash) to evaluate the effects of the stomatognathic system over the postural performances. This second part has only considered patients with a dental malocclusion, analyzing their posture on the stabilometric platform following the Giacomello's Protocol, or else by doing 5 differentiated test of 15 seconds each, carried out in different conditions of occlusion: 1) in rest position, 2) in centric occlusion (habitual), 3) in clenched jaw with habitual occlusion, 4) with occlusal disengage between the arches (e.g. two cotton rolls between the arches), 5) in clenched jaw with cotton rolls in between the arches. From the comparison in between the two groups (with normal occlusion, results are described in the first part, and with malocclusion, results described in this second part), it results that the first group presents statistically better postural performances than the second group, particularly in the rest position conditions, in habitual centric and in clenching in habitual centric. In the test with occlusal disengage the differences reduce themselves, both for the drastic improvement of the second group and for the worsening of the first one. Even in the respect of the tests performed inside the second group, it results that the presence of an occlusal disengage determines a sensible improvement of the postural stability (statistically significant) in relation to the conditions of rest position, centric and clenching in centric: we can deduce the importance of the dental occlusion in the management of the body posture, both in a sense of stabilization (as the patients from group 1) and in a 
sense of destabilization in case of malocclusion (as the patients from group 2).

\section{References}

1. Deli R, Gambone MG, Resta A, Boari A, Grippaudo C (2003) The baropodometric in the evaluation of the relationship between occlusion and posture : a pilot study. Ortognatod Italian 12: 2 .

2. Carra MC, Huynh N, Morton P, Rompré PH, Papadakis A, et al. (2011) Prevalence and risk factors of sleep bruxism and wake-time tooth clenching in a 7- to 17-yr-old population. Eur J Oral Sci 119(5): 386394.

3. Castroflorio T, Falla D, Wang K, Svensson P, Farina D (2011) Effect of experimental jaw-muscle pain on the spatial distribution of surface EMG activity of the human masseter muscle during tooth clenching. J Oral Rehabil 39(2): 81-92.

4. Zhang M, Hasegawa Y, Sakagami J, Ono T, Hori K, et al. (2011) Effects of unilateral jaw clenching on cerebral/systemic circulation and related autonomic nerve activity. Physiol Behav 105(2): 292-297.

5. Farella M, De Oliveira ME, Gallo LM, Läubli T, Tomatis L, et al. (2011) Firing duration of masseter motor units during prolonged low-level contractions. Clin Neurophysiol 122(12): 2433-2440.

6. Rodríguez K, Miralles R, Gutiérrez MF, Santander H, Fuentes A, et al (2011) Influence of jaw clenching and tooth grinding on bilateral sternocleidomastoid EMG activity. Cranio 29(1): 14-22.

7. Sterling M, Chadwick BJ (2010) Psychologic processes in daily life with chronic whiplash: relations of posttraumatic stress symptoms and fear-of-pain to hourly pain and uptime. Clin J Pain. 26(7): 573-582.

8. Madeleine P, Nielsen M, Arendt-Nielsen L (2011) Characterization of postural control deficit in whiplash patients by means of linear and nonlinear analyses-A pilot study. J Electromyogr Kinesiol 21(2): 291297.
9. Côté JN, Patenaude I, St-Onge N, Fung J (2009) Whiplash-associated disorders affect postural reactions to antero-posterior support surface translations during sitting. Gait Posture 29(4): 603-611.

10. Woodhouse A, Stavdahl Ø, Vasseljen O (2010) Irregular head movement patterns in whiplash patients during a trajectory task. Exp Brain Res 201(2): 261-270.

11. Busquet L (2002) Le Catene Muscolari. 1: Marrapese, Roma.

12. Endo K, Suzuki H, Yamamoto K (2008) Consciously postural sway and cervical vertigo after whiplash injury. Spine 33(16): 539-542.

13. Myran R, Hagen K, Svebak S, Nygaard O, Zwart JA (2011) Headache and musculoskeletal complaints among subjects with self reported whiplash injury: the HUNT-2 study. BMC Musculoskelet Disord 12: 129.

14. Phillips LA, Carrol LJ, Cassidy JD, Cote P (2010) Whiplash-associated disorders: who gets depressed? Who stays depressed? Eur Spine J 19(6): 945-956.

15. Holm LW, Carrol LJ, Cassidy DJ, Ahlbom A (2007) Factors influencing neck pain intensity in whiplash-associated disorders in Sweden. Clin J Pain 23(7): 591-597.

16. Ridi R, Saggini R (2003) Equilibrio Corporeo. Edizioni Martina, Bologna, Italy, pp. 296.

17. Baydal-Bertomeu JM, Page AF, Belda-Lois JM, Garrido-Jaen D, Prat JM (2011) Neck motion patterns in whiplash-associated disorders: quantifying variability and spontaneity of movement Clin Biomech 26(1): 29-34.

18. Ivancic PC, Sha D (2010) Comparison of the whiplash injury criteria. Accid Anal Prev 42(1): 56-63.

19. Gagey PM, Weber B (1997) Posturologia. regolazione e perturbazioni della stazione eretta. Marrapese (Eds) Roma.

20. Esposito GM, Messermann JP (1988) Valutazione della relazione esistente tra l'occlusione e la postura. Dent Mod 5: 923-941. 